\title{
Would I Lie to You? How Interaction with Chatbots Induces Dishonesty*
}

\author{
Christian Biener and Aline Waeber
}

July 2021

\begin{abstract}
Is dishonesty more prevalent in interaction with a machine as opposed to a human? We analyze this question using an innovative experimental setup involving the reporting of an unobserved payout-relevant random draw either to a chatbot or another human in a chat interaction while also varying the degree of agency. We find that reporting to a chatbot that is unable to demonstrate agency induces the lowest levels of honesty, whereas reporting to a human, which can demonstrate such agency, generates the highest levels of honesty. We identify a stronger role of social-image concerns and social norms when a person interacts with another human and show that subjects abstain from lying more when they have more time to reflect on their behavior. Our results have implications for designing efficient means of interaction between consumers and organizations in a variety of different contexts in the digital economy. Keywords: lying, honesty, chatbot, communication, lying cost, agency. JEL Codes: C91, D82, D83.
\end{abstract}

*Biener: University of St.Gallen (e-mail: christian.biener@unisg.ch); Waeber: University of St.Gallen (e-mail: aline.waeber@unisg.ch). We thank Kai Barron, Johannes Binswanger, and Matthias Weber as well as the participants of the 2020 Economic Science Association (ESA) Global Meeting, the PhD in Finance (PiF) Seminar and the 9th Research Seminar of the Institute of Insurance Economics at the University of St.Gallen for their helpful comments and discussions. 


\section{Introduction}

Does human economic behavior change when interacting with a machine as opposed to another human being? This a key behavioral question yet to be understood in the context of the vast substitution of humans with automated and autonomous conversational agents (i.e., chatbots) in the digital economy. Recent developments in conversational artificial intelligence $(\mathrm{AI})^{1}$ have enabled the use of such chatbots for automation of service and assistance tasks with significant efficiency gains (for both consumers and organizations) and potential for disruption in various industries such as banking and insurance. ${ }^{2}$

However, there is a potential flip side of replacing human-to-human with human-to-machine interactions: humans might be more inclined to report dishonestly when interacting with a machine as opposed to a human, because lying might simply evoke lower intrinsic costs in the interaction with a machine as opposed to a human. Such intrinsic costs of dishonest behavior have been linked to social-norm compliance (Krupka and Weber 2013), social- (Bursztyn and Jensen 2017) and self-image (Mazar, Amir, and Ariely 2008) concerns, and the velocity of decision making (Shalvi, Eldar, and Bereby-Meyer 2012). A key question in addressing this issue is, whether non-human machines can be designed to be perceived as more human in a sense that it increases the intrinsic costs of dishonest behavior when interacting with them.

We assess these questions in an incentivized behavioral experiment consisting of a one-shot individual reporting of an unobserved random draw via a written online chat interaction and a two-by-two factorial design with randomized assignment, varying the nature of interaction (i.e., human versus machine) and the degree of mind attribution through a signal of agency. To eliminate the confounding effects from differences in presentation and framing, we originate a homogeneous online chat interface that varies only regarding the type of interaction-

\footnotetext{
${ }^{1}$ Research on AI, and indirectly chatbots, dates back to early work by Turing (1950), who developed a measure of a machine's ability to replicate human actions to a degree that is indistinguishable from human behavior. Turing's test inspired many computer scientists and encouraged discussions about machine intelligence, consciousness, and intentionality. In 1964, the AI laboratory at MIT created ELIZA, an interactive computer program that could converse with humans (Weizenbaum 1966). ELIZA creator Joseph Weizenbaum regarded the program as a means to demonstrate the superficiality of human-machine communication, but he was surprised by the number of people attributing human-like feelings to ELIZA. In recent years, the use of chatbots evolved rapidly due to new technologies such as natural language processing, neural networks, and machine learning. A consequence is that the strong increase in chatbot technologies now has the potential to reduce operational costs by replacing human assistants. In fact, a study by Juniper Rse (2019) estimates that the operational costs savings from using chatbots will reach about USD 8 billion in banking and amount to USD 1.3 billion in insurance claims management alone in 2023.

${ }^{2}$ An insurance example is the US digital insurance company Lemonade, which sells insurance policies and settles claims via chatbots or the Swiss insurance company Helvetia, which utilizes chatbots in the claims settling process. Several studies expect this technology to entirely change the means of interactions in both banking (Brewster 2016) and insurance (Hall 2017).
} 
i.e., either an automated machine (i.e., chatbot) or another human. To avoid participants' apprehension about experimenter manipulation, we rely on an external non-physical and verifiable source of uncertainty - decimals of stock index values, which can be easily looked up via a Google widget on the internet. This source of uncertainty at the same time offers the advantages of featuring an objective (uniform) probability distribution that allows us to infer honest reporting at the treatment population level and is available for all web-enabled devices, making it usable for online experimentation. Precluding the concern (and heterogeneity herein) of facing experimenter judgment of reporting behavior as could incur in a standard physical laboraty setting (e.g., at the payout stage), we ensure the anonymity and nonobservability of participants when conducting the experiments not in the laboratory, but at home in an online setting. This latter design feature mimics real-world settings of chatbot interactions and thus magnifies external validity.

We observe a material and significant amount of dishonest reporting behavior in our data, unconditional on treatment assignment. The average reported outcome is 6.5 and the distribution of reports is highly skewed towards the high payoff outcomes. This result is in contrast to the uniform distribution across all possible values between 0 and 9 that would generate an expected average reported outcome of 4.5 under full honesty. Our exogenous variations generate variation in reporting behavior commensurate with our hypotheses. While we find that reporting to a chatbot induces the lowest levels of honesty, we see that endowing the human chat interaction with the indication of human agency (i.e., by signaling mind via a variation in the interaction content) causes a significant increase in honesty. In contrast, endowing the chatbot with an indication of agency does not induce more honest behavior. We also show that values of perceived agency indeed decline in the expected order: a human with agency, a human without agency, a chatbot with agency, and a chatbot without agency. A human that can signal agency in a chat communication is thus being reported to most honestly, and the joint effect of replacing a human with a machine and removing agency is an increase of 17 percent in reported outcomes (i.e., 0.43 standard deviations).

Our analysis of pathways further supports the expected role of social- and self-image concerns, social norms, and velocity of decision making. More precisely, we find that individuals pay less attention to their social image when they interact with a machine principal (even when this machine principal signals agency) as opposed to a human. Furthermore, an experiment in which participants' beliefs about other's behavior were elicited shows that, aside from the higher prevalence of social-image concerns under then human condition, differences in perceived norms might play a role in explaining behavior. Finally, our results suggest that a passive interaction via a standard online form as opposed to a conversational chat 
interaction could achieve the same levels of honesty as the human-to-human chat interaction.

Considering the economic costs caused by dishonest behavior, reducing dishonest behavior effectively is of great relevance to policy makers and the economy in general (Mazar and Ariely 2006). Dishonesty poses a severe negative externality to markets, generating severe costs of doing business, which can ultimately lead to the unraveling of markets. An example of everyday deception is insurance fraud, which is estimated to be exceeding USD 40 billion per year in the US non-health insurance market alone, increasing premiums for an average family to between USD 400 and USD 700 annually (FBI 2020). The following puts our results into perspective: if today's human-dominated claims reporting processes are replaced by chatbots, based on our results, we could expect an increase in fraudulent claims of roughly USD 7 billion in the US non-health insurance market alone. Comparable levels of dishonesty can be observed in non-market settings such as tax reporting, ${ }^{3}$ thus substantially distorting measures of inequality (Alstadsæter, Johannesen, and Zucman 2019). This implies the crucial relevance of understanding such behavioral implications of technological innovations for markets and society as a whole.

The remainder of this paper proceeds as follows. In Section 2, we provide a broad overview of the related literature and derive our hypotheses. Section 3 provides an introduction to the experimental setting, including information on treatment variation and procedures. In Section 4, we present the results, and in Section 5 we conclude.

\section{Literature and hypotheses}

Our work first and foremost contributes to a new field of empirical research focused on understanding the differences between human-to-human and human-to-machine interactions. Nested in this new stream of literature is the fundamental question of the impact of human-tomachine interactions on honest behavior in market transactions. Standard economic theory suggests that individuals would always lie if benefits (i.e., monetary gains) are sufficiently high to cover the costs of dishonest behavior (i.e., punishment). However, much of the literature has shown that individuals are often willing to forego financial benefits to behave honestly in such situations (Abeler, Becker, and Falk 2014; Cappelen, Sørensen, and Tungodden 2013; Erat and Gneezy 2012; Fischbacher and Föllmi-Heusi 2013; Gneezy 2005; Gneezy, Kajackaite, and Sobel 2018; Mazar, Amir, and Ariely 2008). In the context of human-to-

\footnotetext{
${ }^{3}$ A recent Internal Revenue Service (IRS) study estimates that the tax gap (i.e, the difference between what the IRS estimates taxpayers should pay and what they actually pay) is USD 441 billion annually for the 2011-2013 timeframe (Internal Revenue Service 2019).
} 
machine interactions, there is some empirical evidence that some differences might arise in honest behavior, conditional on whether a human interacts with another human or with a machine. Hoegen et al. (2015), for example, compare the behavior of people interacting with other humans in face-to-face interactions with their behavior when interacting with virtual humans. They find that subjects are more willing to steal when interacting with virtual humans. Recently, Maréchal, Cohn, and Gesche (2020) show that lying is more prevalent when subjects interact with a machine in the form of a voice response system with pre-recorded voice messages as opposed to a call with another human. In this paper, we focus on a recent key technological innovation - artificial conversational agents or chatbots - now broadly applied by corporations around the globe.

Hypothesis 1 (Human versus machine): We expect participants to be less honest in an interaction with a machine as opposed to an interaction with another human.

Despite recent advancements in chatbot technology, no artificial intelligence has yet been developed that has the basic features of what psychologists call a "theory of mind" (Astington 1994; Baron-Cohen 2000). A theory of mind is defined as the human ability to attribute mental states such as beliefs, desires, goals, and intentions to others as well as the ability to deceive others (Rabinowitz et al. 2018). This ability enables humans to conceive of the different mental states that other people demonstrate. Such an ability is thus essential for human cognition and social interactions. For example, theory of mind enables us to understand the meaning behind an ironic remark. Evidence on the theory of mind concept mainly originates from the field of cognitive neuroscience. In an early contribution to neuroeconomics, McCabe et al. (2001) compare neural activity in a trust game with both human and computer counterparts. They find significant increases in activation in medial prefrontal brain regions among cooperators in human-to-human interactions compared to human-to-machine interactions. This brain region is thus involved in integrating theory-ofmind processing with cooperative behavior. However, further studies reveal mind attribution of humans when interacting with machines. Tanibe, Hashimoto, and Karasawa (2017) show that imagining a situation in which people treat a robot in a good way made them more willing to attribute a mind to it, and increased mind attribution led to more positive attitudes toward the robot. Furthermore, Nass and Moon (2000) show that it is possible for humans to ascribe human-like attributes to machines. They argue that such behavior is due to "mindless overusage" of human social categories and "overlearned" social behaviors. In a laboratory experiment, Reeves and Nass (1996) find that humans are as polite to computers as they are to humans. Similarly, Fogg and Nass (1997) reveal that individuals apply gender stereotypes to computers even though they are aware of the machine narrator. 
Hypothesis 2 (Signaling of agency): We expect the endowment of the human counterpart with an indication of agency (i.e., by signaling mind via a variation in the interaction content) to increase honest behavior, while an indication of agency of the machine counterpart is not expected to cause this effect, because humans expect other humans (but not machines) to possess a mind.

We explore a range of further pathways that could determine honest behavior in our setting. The act of lying violates an individual's self-concept as an honest person (Mazar, Amir, and Ariely 2008; Shalvi, Eldar, and Bereby-Meyer 2012), and recent evidence suggests that individuals also experience social-image concerns as a result of their dishonesty (Abeler, Nosenzo, and Raymond 2019; Khalmetski and Sliwka 2019). Recent theoretical models combine both a desire to appear honest with a desire to be honest in the utility function (Abeler, Nosenzo, and Raymond 2019; Khalmetski and Sliwka 2019). People thus not only consider the expected monetary gains from lying, the probability of being caught, and the potential punishment, but also care about not being perceived as a liar by themselves but also by the receiver of the message at the time of communication. Following this literature, we expect that some participants incur social- or self-image costs of lying as they experience a direct disutility from falsely reporting private information. In the interaction with a machine principal, such social- or self-image costs of lying play a less important role because participants feel less comfortable reporting a lie to a human as opposed to a machine. We thus expect the share of participants who lie maximally (i.e., report high outcomes) to be higher when reporting to a machine. Similarly, we expect the share of partial liars (i.e., reporting more credible outcomes) to be higher when subjects report to a human principal.

A large body of literature suggests that deception is cognitively more demanding than responding honestly, and thus, honesty is considered the behavioral default (Foerster et al. 2013). However, other studies have reported the opposite (Shalvi, Eldar, and Bereby-Meyer 2012; Piovesan and Wengström 2009). More precisely, Shalvi, Eldar, and Bereby-Meyer (2012) show that lying is an initial, automatic tendency that is overcome only if sufficient time to deliberate is available and if dishonest behavior cannot be justified. This is supported by earlier findings in neuropsychological research showing that the right dorsolateral prefrontal cortex, a brain area involved in executive control, is associated with overriding selfish impulses in economic decisions (Knoch et al. 2006). This area, together with two other brain areas associated with self-control, is activated when individuals make an effort to forgo lying (Greene and Paxton 2009). In line with the above findings, Piovesan and Wengström (2009) find that faster participants make more egoistic choices in a dictator game. Thus, cooperation involves a conflict that implies an extra cognitive load and is thus slower. Previous research 
also finds an inverted-U relationship between response time and cooperation (Evans, Dillon, and Rand 2015). Following a recent meta-analysis identifying honesty as deliberative (Köbis et al. 2019), we anticipate decision times and dishonesty to be negatively correlated.

Gneezy, Imas, and Madarász (2014) show that the intrinsic costs of lying might originate from a conditional aversion to lying. We thus predict that participants feel less guilty when they lie to a machine principal. Lastly, we expect differences in perceived norms (i.e., beliefs of others' behavior) to explain differences in dishonest behavior.

Individuals often do not take full advantage of a lying opportunity (Mazar, Amir, and Ariely 2008; Fischbacher and Föllmi-Heusi 2013), which can be explained by the presence of an internalized honesty norm that creates intrinsic costs of lying (Fischbacher and Föllmi-Heusi 2013; Kocher, Schudy, and Spantig 2017; Cohn, Fehr, and Marechal 2014; Krupka and Weber 2013). We expect that the presence of a machine as opposed to a human interaction changes the perception of the norm.

\section{Experimental design}

Our design follows the fundamental ideas of standard experimental setups to infer dishonest behavior (e.g., Fischbacher and Föllmi-Heusi 2013) by asking participants to report a randomly generated number, which is unobservable by the experimenter and which determines the payoff for participating in a survey. The context of the survey was framed in the health and risk domain, including questions about health status and health behavior as well as questions about the propensity to take risks in different areas.

In contrast to the use of physical sources of uncertainty such as a die (Fischbacher and Föllmi-Heusi 2013) or a coin (Maréchal, Cohn, and Gesche 2020), which can be problematic for online experimentation, we use the value of a stock market index to generate random numbers. In particular, the random number is generated by the second decimal place of either the Swiss Market Index (SMI) or the DAX Performance Index (DAX) at a particular point in time - the reported outcome equals the payoff in Swiss Francs (CHF). Participants in the experiment are asked to look up the value of their respective stock index of choice in a Google widget, showing either the SMI or the DAX (see Figure 1 for an example of the SMI). We then ask them to report the observed random draw via a written chat communication on a computer screen. We emphasize that we will not learn the participants' choice of index, lending further credibility to the unobservability of the source of uncertainty, which 
is important to avoid reputation and strategic concerns. ${ }^{4}$ Because the payoff participants receive for participation depends on the reported outcome, there is a clear incentive to report higher outcomes.

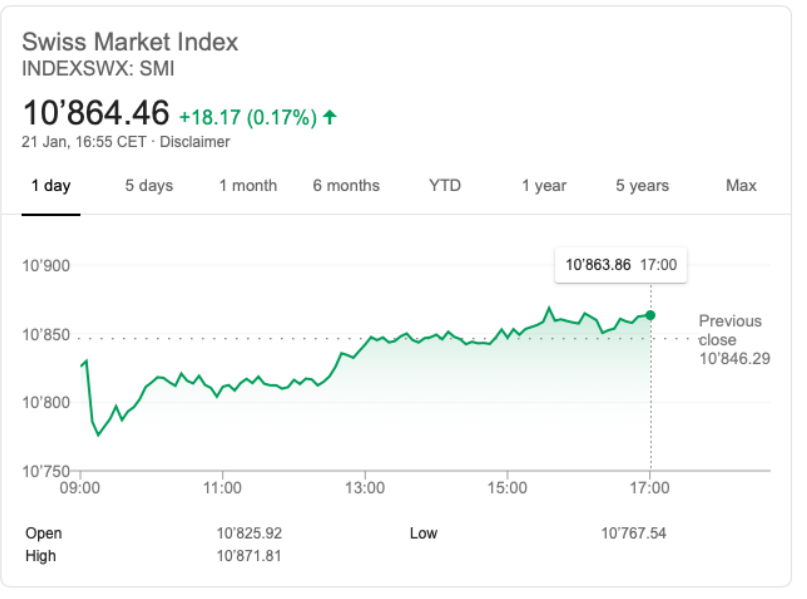

Figure 1: Google widget for SMI

By design of the experiment, we cannot detect dishonesty at the individual level, but, because we know the empirically realized distribution of values, ${ }^{5}$ we can infer dishonesty for different subpopulations along our dimension of treatment variation.

We follow Gneezy, Imas, and Madarász (2014) and further administer an incentivized measure of guilt to assess whether and how significantly the feeling of guilt plays a differential role between human and machine interactions. Here, we ask participants how much of their proceeds from the experiment they would like to donate to UNICEF (i.e., an amount in the range [CHF 0, $\mathrm{CHF} 1]$ in ten centime increments). We use this range so that the donation amount is relatively small compared to the amount that can be gained through dishonesty.

\footnotetext{
${ }^{4}$ Using an online experiment, we seek to eliminate fears of detection, punishment, or reputational concerns (e.g., when interacting with the lab personnel). To test, whether fear of detection plays a role, we elicit subjects' risk attitudes to test whether there is a relationship between risk aversion and dishonest behavior. If the fear of detection is a relevant issue in our design, we would expect that more risk averse subjects are less dishonest. We further include an interaction term between risk aversion and the machine dummy to see whether subjects are more worried about getting caught in the interaction with a human principal. As shown in Table A1 of the appendix, subjects' risk aversion does not significantly decrease reported outcomes. We additionally find that dishonesty is not significantly different under either condition (i.e., human or machine principal). It can thus be concluded that punishment or reputation concerns do not play a role in our experimental design.

${ }^{5}$ The distribution of second decimals in the range of $[0,9]$ of the DAX and SMI stock index is not only known approximately but exactly, as we look up the data in 5-minute intervals at a later point in time in the respective Google widgets. It is important to emphasize that we cannot observe the same data as the participants do during the time of the experiment. We show in Figure A1 of the appendix that these values are indeed equally distributed.
} 
We further elicit the subjects' risk attitudes using the survey questions developed by Dohmen et al. (2011). ${ }^{6}$

\subsection{Treatment variations}

We implement four treatments in a between-subject design as shown in Table 1. Table 2 further shows selected communication elements to illustrate the similarities and differences in the treatment conditions. In order to isolate the impact of the type of principal and the role of agency on dishonest behavior, we follow a standard two-by-two factorial design, varying the dimensions of (1) human versus chatbot and (2) agency versus no agency. While under the human conditions (i.e., $C_{H U}, C_{H U: A G E N C Y}$ ), participants interact with a student assistant, participants interact with an automated chatbot under the bot conditions (i.e., $\left.C_{B O}, C_{B O: A G E N C Y}\right)$. The chatbot was programmed to be gender and age neutral.

Table 1: Treatment conditions

\begin{tabular}{cc|cc} 
& & \multicolumn{2}{|c}{ Principal type } \\
& & Human & Bot \\
\hline \multirow{2}{*}{ Agency } & No & $C_{H U}$ & $C_{B O}$ \\
& Yes & $C_{H U: A G E N C Y}$ & $C_{B O: A G E N C Y}$ \\
\hline
\end{tabular}

We further vary the dimension of agency (i.e., $C_{H U: A G E N C Y}, C_{B O: A G E N C Y}$ ). Under these conditions, we facilitate mind attribution among agents toward the principal. Gray, Gray, and Wegner (2007) show that people perceive mind in two dimensions: the perceived capacity to sense and feel (i.e., experience) ${ }^{7}$ and the perceived capacity to act and do (i.e., agency). ${ }^{8}$ Adult humans are seen as capable of both agency and experience, whereas machines are seen as capable of mainly agency. However, a machine's ability to think is often quite domain specific. Considering the full spectrum of agency, machines are perceived as having less agency than adult humans (Gray and Wegner 2012). Endowing computers with experience can lead to a phenomenon that has been described as "the uncanny valley", referring to the increasing discomfort humans feel in encountering robots when they appear to be humanminded. Through an experiment, Gray and Wegner (2012) find that a machine capable of

\footnotetext{
${ }^{6}$ Precisely, we asked subjects, "How do you see yourself: Are you generally a person who is fully prepared to take risks or do you avoid taking risks" using an 11-point Likert scale ranging from "not at all willing to take risks" to "very willing to take risks".

${ }^{7}$ Attributed to hunger, fear, pain, pleasure, rage, desire, personality, consciousness, pride, embarrassment, and joy.

${ }^{8}$ Including the capacities of self-control, morality, memory, emotion recognition, planning, communication, and thought.
} 
experience causes feelings of unease, even without a humanlike appearance. We thus focus on perceived agency here.

More specifically, in $C_{B O: A G E N C Y}$, the chatbot was programmed to have a discussion on its own capabilities, showing that it can communicate and think (e.g., that it can process a lot of data and interactions at the same time and that it can mimic conversation-like interactions). Similarly, in $C_{H U: A G E N C Y}$, the student assistant reflects on his capabilities (e.g., that he/she is providing research assistance). The chat communication software allows for both human and machine interaction through the same chat interface, allowing us to avoid differences in format. ${ }^{9}$

Table 2: Exemplary conversational interactions conditional on treatment

\begin{tabular}{|c|c|c|}
\hline & \multicolumn{2}{|c|}{ Principal type } \\
\hline & Human & Bot \\
\hline Agency No & $\begin{array}{l}\text { Hello! I'm a student assistant, helping the } \\
\text { experimenters to collect your responses for } \\
\text { this survey. Are you ready? }\end{array}$ & $\begin{array}{l}\text { Hello! I'm an automated chatbot, the } \\
\text { experimenters programmed to collect your } \\
\text { responses for this survey. Are you ready? }\end{array}$ \\
\hline Agency Yes & $\begin{array}{l}\text { I'm a talented student, which is, I believe, } \\
\text { the reason, I'm being involved in this } \\
\text { survey. I also have good communication } \\
\text { skills, helping me to understand others, } \\
\text { and to be understood. }\end{array}$ & $\begin{array}{l}\text { I can process a lot of data and interactions } \\
\text { at the same time, which is, I believe, the } \\
\text { reason, I'm being involved in this survey. } \\
\text { At the same time, I can mimic } \\
\text { conversation-like interactions, which are } \\
\text { closer to human communication than } \\
\text { online forms. }\end{array}$ \\
\hline
\end{tabular}

\subsection{Procedures}

We recruited participants from the participant pool of the behavioral lab at the University of St.Gallen. Because some of the treatment conditions of the experiment rely on human interaction, and our design requires that we conduct sessions during the trading hours of the SMI and DAX, participants were asked to select a time slot before taking part in the study. The web-link to the study was sent out in a separate e-mail shortly before the session started. We explicitly asked participants to make sure that they were in a quiet place without any distractions when starting the experiment.

During the experiment, we informed participants that the experimental data is anonymized

\footnotetext{
${ }^{9}$ Under the human conditions $C_{H U}$ and $C_{H U: A G E N C Y}$, the conversation is always led by a student assistant interacting with participants.
} 
and treated confidentially. Participants first received instructions of the experiment via the experimental software oTree (Chen, Schonger, and Wickens 2016) and were randomly assigned to one of the four conditions, assuring equal distribution of treatments within one experimental session. We informed participants about the nature of the principal (i.e., chatbot or human), conditional on treatment assignment. The student assistants interacting with participants under the human conditions strictly followed a conversational protocol, ensuring that the content of conversations is comparable between human and bot conditions (see Table A2 of the appendix for the complete conversational protocol). Figure 2 shows the chat inferface that appeared on the main experiment page in the case of the machine interaction treatments. To ensure credibility of the human nature of the principal with regard to response times, we manually lagged the conversational input of the human as opposed to the bot principal slightly. The average experiment duration (in minutes) is 7.06 for $C_{B O}, 8.16$ for $C_{B O: A G E N C Y}, 11.04$ for $C_{H U}$ and $13.91 C_{H U: A G E N C Y}$. We carefully control for the potential role of these differences in our later analyses and find no meaningful effect. Participants were compensated with a fixed participation fee of $\mathrm{CHF}$ 3, plus an additional payoff that was conditional on the reported random draw (i.e., ranging between CHF 0 and CHF 9). Payments were sent to the participants' bank accounts the evening of the day of participation. We asked participants for their bank account information on a separate website connected to a separate database that we could not link with the experimental data. 


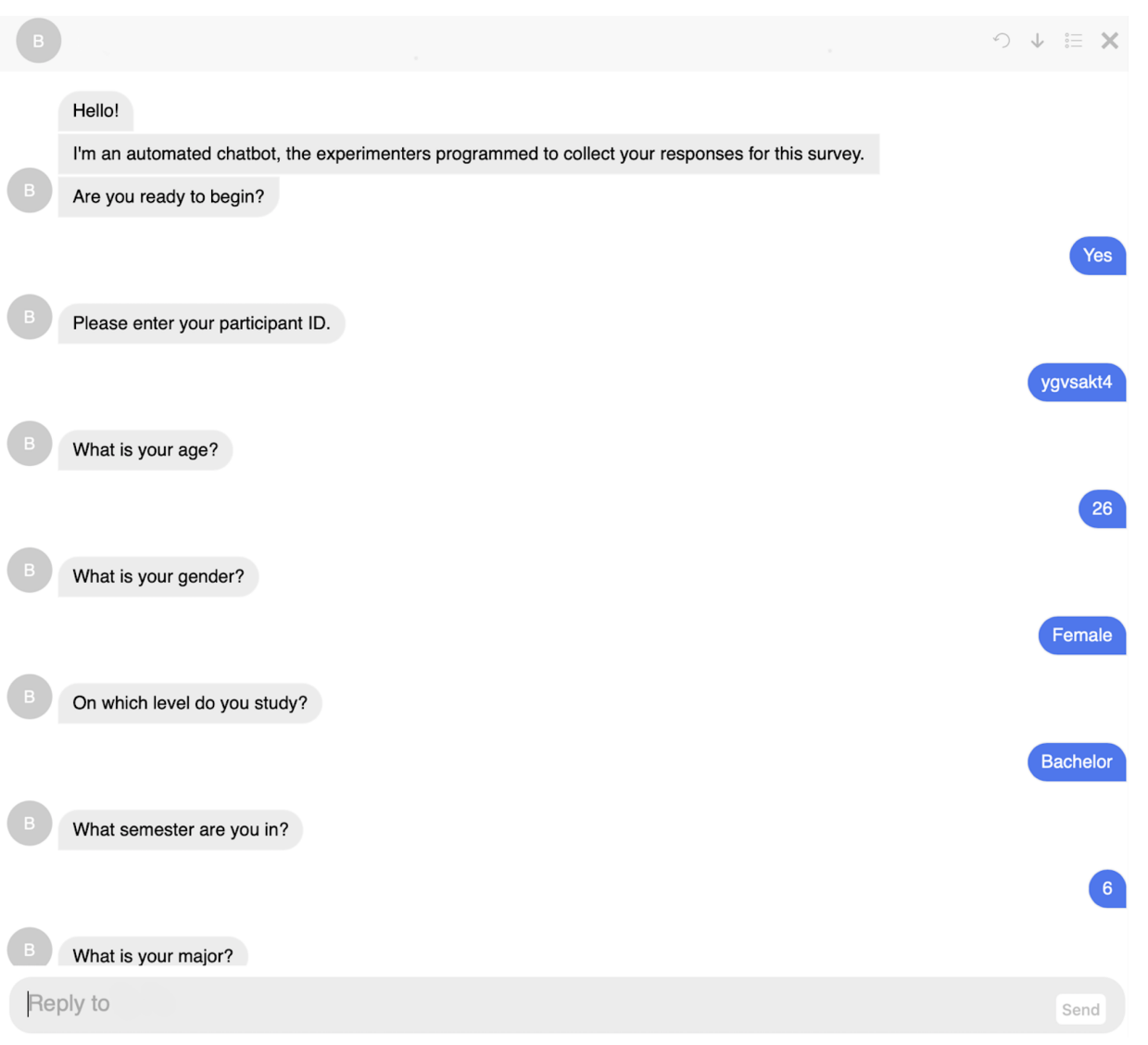

Figure 2: Chat interface

\subsection{Summary statistics}

A total of 381 participants with an average age of 23.5 took part in our experiment. ${ }^{10}$ The sample has slightly more male participants (59 percent) as opposed female participants (41 percent). About 56 percent of respondents reported to have between CHF 500 and CHF 1,499 at their disposal per month, representing the largest income category in our sample. When asked about their sources of income, 62 percent of respondents indicated their family and 29 percent reported their job as their main source of income. The majority of the participants (27 percent) had a study major in Management. Table 3 provides summary statistics and balancing tests of our control variables. Except for marginally significantly different income sources under $C_{B O}$, our sample appears balanced across treatment conditions. This is expected due to randomized treatment assignment.

\footnotetext{
${ }^{10}$ Using a previous results on human-human versus human-machine interactions on dishonesty in Maréchal, Cohn, and Gesche (2020) as a benchmark, we conducted a power calculation based on a two-sided two-sample means test. Power was set to 0.8 , while alpha was set to 0.05 . The resulting sample size yielded about 100 subjects per group.
} 
Table 3: Summary statistics and balancing tests

\begin{tabular}{lccccc}
\hline \hline & Gender & Age & Income & Income Source & Major \\
& $(1)$ & $(2)$ & $(3)$ & $(4)$ & $(5)$ \\
\hline$C_{B O: A G E N C Y}$ & 0.028 & 0.795 & 0.028 & 0.060 & -0.022 \\
& $(0.072)$ & $(0.532)$ & $(0.072)$ & $(0.070)$ & $(0.065)$ \\
$C_{H U}$ & 0.064 & -0.111 & 0.075 & 0.054 & -0.077 \\
& $(0.071)$ & $(0.531)$ & $(0.072)$ & $(0.070)$ & $(0.064)$ \\
$C_{B O}$ & 0.033 & 0.416 & -0.062 & $0.149^{* *}$ & -0.088 \\
& $(0.071)$ & $(0.531)$ & $(0.072)$ & $(0.070)$ & $(0.064)$ \\
Constant & $0.557^{* * *}$ & $23.247^{* * *}$ & $0.546^{* * *}$ & $0.557^{* * *}$ & $0.320^{* * *}$ \\
& $(0.050)$ & $(0.373)$ & $(0.050)$ & $(0.049)$ & $(0.045)$ \\
\hline Mean & 0.59 & 23.52 & 0.56 & 0.62 & 0.27 \\
Std. deviation & 0.49 & 3.68 & 0.5 & 0.49 & 0.45 \\
Observations & 381 & 381 & 381 & 381 & 381 \\
\hline \hline
\end{tabular}

Notes: The table shows OLS estimates of differences between the treatment conditions and the base condition $C_{H U: A G E N C Y}$. The constant term represents the average outcomes for the subsample assigned to $C_{H U: A G E N C Y}$. For ease of interpretation, we recoded our categorial variables into binary variables (i.e., dummy coding) in each case assigning 1 to to largest category. Gender is a dummy variable that takes on the value 1 when the participant is male. Income is a dummy variable that takes on the value of 1 if the participant has between CHF 500 to 1,499 at his/her disposal per month. Income source is a dummy taking on the value of 1 if their main source of income is their family. Major is a dummy taking on the value of 1 if the participant's major is Management. *** Significant at the 1 percent level. ** Significant at the 5 percent level. * Significant at the 10 percent level. 


\section{Results}

Figure 3 shows the distribution of the reported outcomes in the total sample, unconditional on treatment assignment. We find that the average reported outcome is 6.5 and that the distribution of outcomes is highly negatively skewed (i.e., towards the high payoff outcomes). This suggests that participants systematically report higher outcomes than the ones they had actually seen, which is in contrast to the uniform distribution across all possible values between 0 and 9 that would generate an expected average outcome of 4.5 under full honesty (i.e., a one-sided Kolmogorov-Smirnov test of equality with the uniform distribution gives $p$-value $<0.001)$. Following Fischbacher and Föllmi-Heusi (2013) to characterize our sample population, we estimate the fraction of honest participants (i.e., reporting an outcome of 0 ) to be 26 percent and the fraction of income maximizers (i.e., reporting an outcome of 9) to be 14 percent. ${ }^{11}$

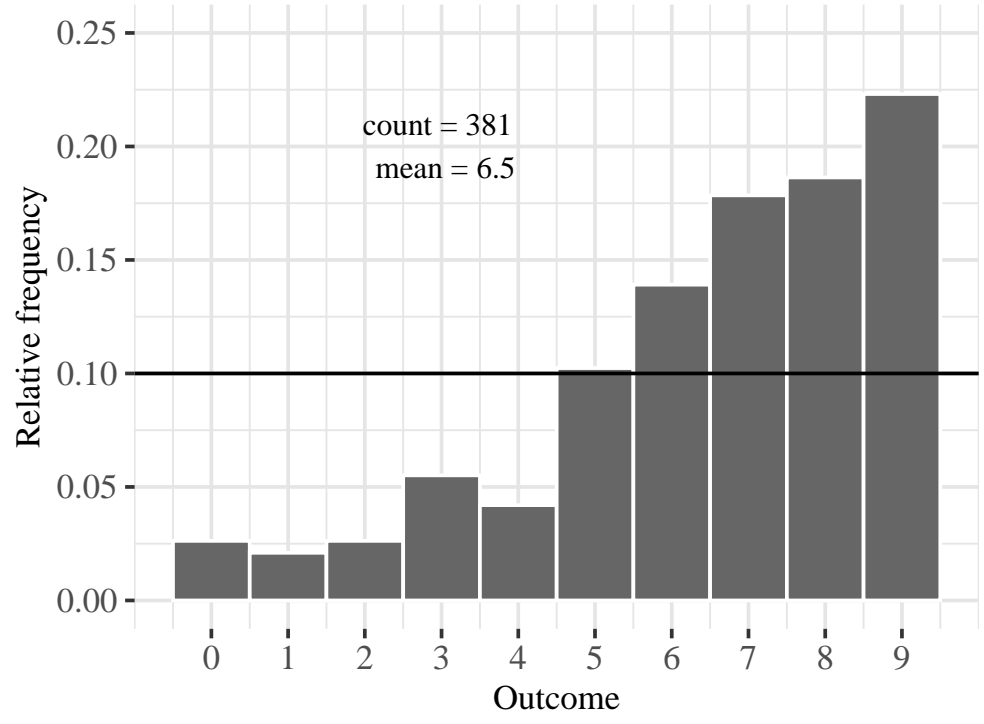

Figure 3: Reports of random draws in total sample

Note: The figure shows the distribution of reported random draws unconditional on treatment assignment. The black solid line depicts the uniform distribution expected under truthful reporting.

The distribution of reported outcomes conditional on treatment assignment in Figure 4 and the estimates of the average size of treatment effects shown in Table 4 provide support for our two main hypotheses. First, we find that replacing the human principal by a machine principal whilst both exhibit agency (i.e., $C_{H U: A G E N C Y}$ versus $C_{B O: A G E N C Y}$ ) induces an

\footnotetext{
${ }^{11}$ Fischbacher and Föllmi-Heusi (2013) estimate the percentage of honest people to be as large as 39 percent and the percentage of participants acting as income maximizers to be 22 percent at maximum.
} 
average increase of $0.843(p$-value $=0.012)$ in reported outcomes. Second, we observe that removing the signaling of agency in the human-to-human interaction (i.e., $C_{H U: A G E N C Y}$ versus $\left.C_{H U}\right)$ increases average reported outcomes by 0.674 ( $p$-value $\left.=0.043\right)$. Third, the combined effect of replacing the human principal by a machine principal and eliminating the signaling of agency (i.e., $C_{H U: A G E N C Y}$ versus $C_{B O}$ ) increases average reported outcomes by $0.991\left(p\right.$-value=0.003) ${ }^{12}$ Fourth, endowing the machine principal with agency does not significantly affect behavior $(p$-value $=0.658)$.
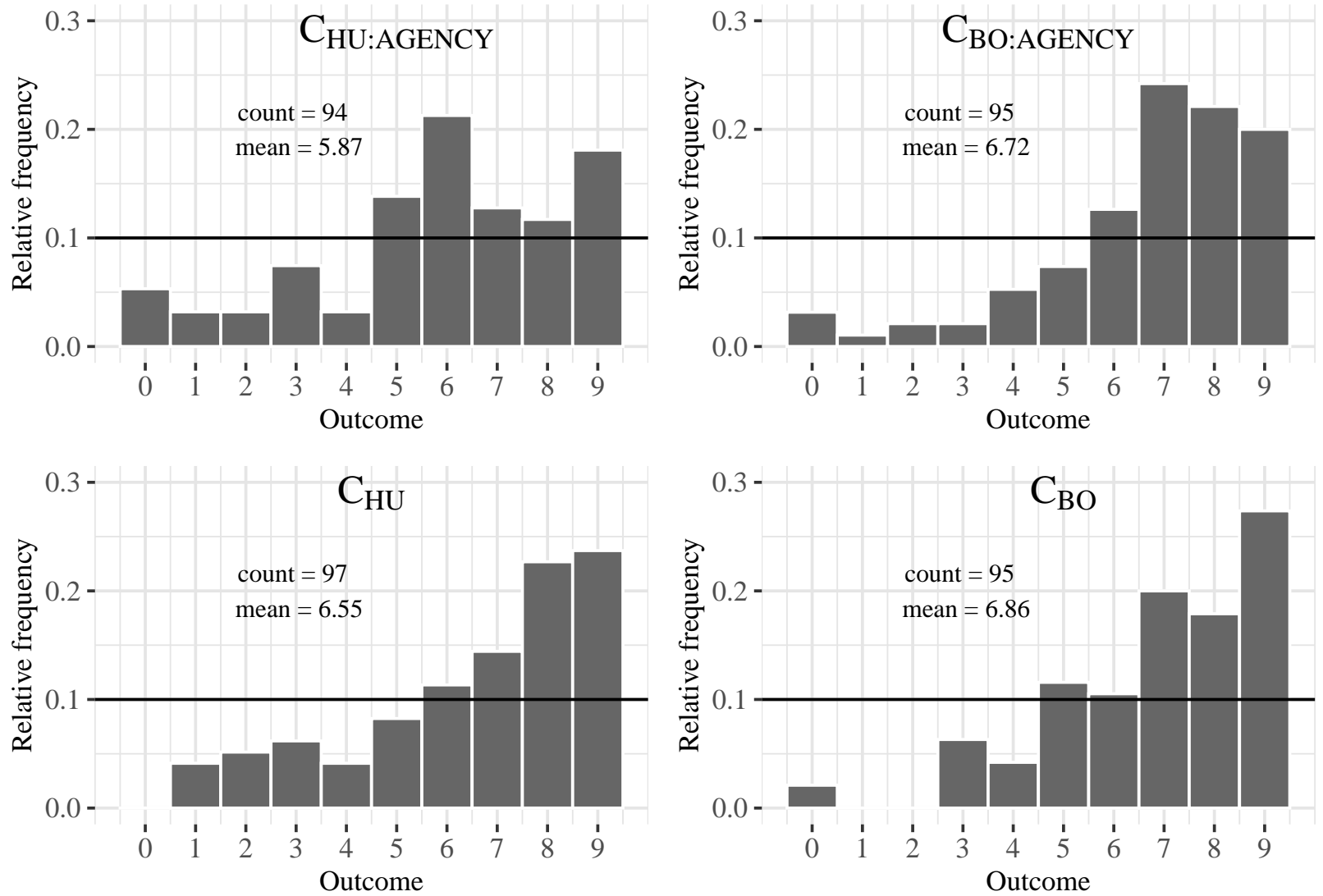

Figure 4: Reports of random draws

Note: The figures show the distributions of reported random draws conditional on treatment assignment. The black solid lines depict the uniform distribution expected under truthful reporting.

As a result of these findings, we infer that both the human-type of interaction as well as the signaling of agency is important to induce honest behavior and that the signaling of agency is not effective, given the machine nature of the principal, while it is effective, given the human nature of the principal.

\footnotetext{
${ }^{12}$ A nonparametric Wilcoxon test confirms the significance of this difference $(p$-value $=0.006)$.
} 
Table 4: Impact of machine nature and agency on reported outcomes

\begin{tabular}{lcc}
\hline \hline & $(1)$ & $(2)$ \\
\hline Constant & $5.872^{* * *}$ & $6.125^{* * *}$ \\
& $(0.236)$ & $(0.446)$ \\
$C_{B O: A G E N C Y}$ & $0.843^{* *}$ & $0.739^{* *}$ \\
& $(0.333)$ & $(0.369)$ \\
$C_{H U}$ & $0.674^{* *}$ & $0.622^{*}$ \\
& $(0.332)$ & $(0.341)$ \\
$C_{B O}$ & $0.991^{* * *}$ & $0.865^{* *}$ \\
& $(0.333)$ & $(0.383)$ \\
Duration & & -0.018 \\
& & $(0.027)$ \\
\hline Observations & 381 & 380 \\
\hline \hline
\end{tabular}

\subsection{Pathways}

\section{Perceived agency}

An important part of the human perception of mind is agency (i.e., the perceived capacity to act and do), and we find that our treatment variation aimed at indicating agency increases people's honest outcome reporting as hypothesized, only in an interaction with another human (see previous section). While this is a central finding, the treatment effect could in principle be driven by mechanisms other than the perception of agency. ${ }^{13}$ To test for the validity of attributing the treatment effect to a variation in mind perception, participants were asked to rate the extent to which they perceived their respective counterpart to have agency subsequent to the interaction and to reporting the outcome in the experiment. We measure agency using four survey items. ${ }^{14}$

\footnotetext{
${ }^{13}$ For example, the specifics of the text elements used to signal agency might have triggered differential reactions not related to agency.

${ }^{14}$ Following Gray, Gray, and Wegner (2007), participants were asked (1) "To what extent is the counterpart capable of having experiences and being aware of things?", (2) "To what extent is the counterpart capable of understanding how others are feeling?", (3) "To what extent is the counterpart capable of telling right from wrong and trying to do the right thing?", and (4) "To what extent is the counterpart capable of making plans and working toward a goal?" All questions were answered on a five-point Likert scale, ranging from not
} 


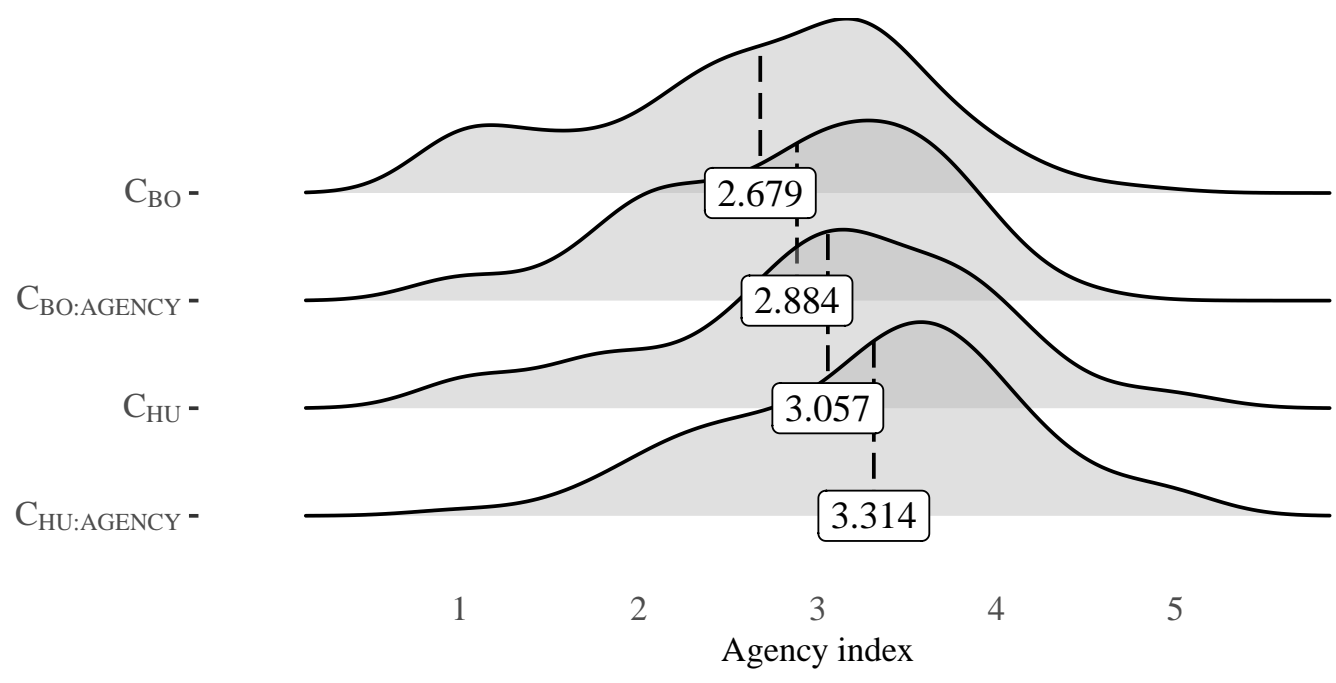

Figure 5: Agency index across conditions

Note: The figure shows kernel density estimates of perceived agency conditional on treatment assignment. Dashed lines indicate mean values.

Figure 5 shows kernel density estimates of perceived agency conditional on treatment assignment. Overall, we find that mean perceived agency is significantly higher under the human conditions as opposed to the machine conditions ( $p$-value $<0.001)$, indicating that humans understand other humans to possess a mind but not machines. As to our intended exogenous variation in agency, we find that indeed it increases perceived agency, but only under the human condition. Mean perceived agency in $C_{H U: A G E N C Y}(M=3.314)$ is significantly higher $(p$-value $=0.039)$ compared to $C_{H U}(M=3.057)$. Under the machine condition, the agency treatment does not impact perceived agency significantly ( $p$-value $=0.096)$. Chatting with a bot thus can -if at all - trigger mind perception only to a limited extent.

\section{Social-image concerns}

In the next step, we aim to examine whether the observed treatment differences in honest behavior can be explained by differences in social-image concerns. The presence of a human principal might cause participants to care more about their social image and thus, they would feel less comfortable telling a lie during the human interaction. To quantify the influence of social-image concerns on dishonesty as well as heterogeneity conditional on treatment assignment, we follow Maréchal, Cohn, and Gesche (2020) and differentiate the reported outcomes into high (i.e., seven, eight, or nine) - and therefore suspicious - and credible (i.e., five or six).

capable at all (1) to very capable (5). We averaged the final answers to construct an agency index. 
Table 5 shows Probit estimates of the treatment effects on reporting high (Column (1)) and medium (Column (2)) outcomes, respectively. First, we find that subjects who communicate with a machine principal exhibiting agency are significantly more likely to report high outcomes $(+22.3$ percentage points, $p$-value $<0.001)$ as opposed to subjects who communicate with a human principal exhibiting agency (i.e., $C_{H U: A G E N C Y}$ versus $C_{B O: A G E N C Y}$ ). Second, we observe that when subjects are exposed to a human unable to signal agency (i.e., $C_{H U: A G E N C Y}$ versus $C_{H U}$ ), the probability to report a high outcome increases by 17.3 percentage points $(p$-value $=0.008)$. Third, replacing the human principal by a machine principal and eliminating the signaling of agency (i.e., $C_{H U: A G E N C Y}$ versus $C_{B O}$ ) increases the probability to report a high, and therefore suspicious, outcome by 21.3 percentage points ( $p$-value $=0.001)$. Finally, solely endowing the machine principal with agency does not significantly affect the probability to report a high outcome $(p$-value $=0.879)$.

The treatment effects on reporting medium and thus more credible outcomes suggest that, by and large, credible reportings are substituted by high suspicious reportings when either withdrawing agency (-13.4 percentage points in credible outcomes, $p$-value $=0.008$ ) or replacing the human counterpart with a machine (-13 percentage points in credible outcomes, $p$-value $<0.01)$ or both $(-11.1$ percentage pointsin credible outcomes, $p$-value $=0.031)$. In summary, our results indicate that individuals pay less attention to their social image when they are interacting with a machine principal (even when this machine principal signals agency) as opposed to a human.

Table 5: Impact of machine nature and agency on reporting high/medium outcomes

\begin{tabular}{lcc}
\hline \hline & High & Medium \\
& $(1)$ & $(2)$ \\
\hline$C_{B O: A G E N C Y}$ & $0.223^{* * *}$ & $-0.130^{* * *}$ \\
& $(0.063)$ & $(0.050)$ \\
$C_{H U}$ & $0.173^{* * *}$ & $-0.134^{* * *}$ \\
& $(0.065)$ & $(0.050)$ \\
$C_{B O}$ & $0.213^{* * *}$ & $-0.111^{* *}$ \\
& $(0.063)$ & $(0.051)$ \\
\hline Observations & 381 & 381 \\
\hline \hline
\end{tabular}

Notes: The table shows Probit estimates (marginal effects) of the treatment effects on reporting high (i.e., $>6$ ) and medium (i.e., 5, 6) outcomes. The reference category is $C_{H U: A G E N C Y}$. *** Significant at the 1 percent level. ** Significant at the 5 percent level. * Significant at the 10 percent level. 


\section{The cognitive load of honesty}

The time to reflect about the honesty of one's behavior has been documented to affect decisions (e.g., Köbis et al. 2019). In our experiment, we observe the time difference (in seconds) between the instruction to look up the random number and the actual reporting of the outcome. Table 6 shows the linear regression point estimates of this relationship, also including the squared response time as an independent variable to account for a potentially U-shaped relationship. Our results confirm what Shalvi, Eldar, and Bereby-Meyer (2012) had indicated, that participants who take longer to report are more honest. More specifically, we report a significant and negative relationship between response time and lying with a one-second increase in the time to report changing the reported outcome by $-0.013(p$ value $=0.028$. Thus, honesty seems to be cognitively more demanding than acting dishonestly. Contrary to the apparent visual impression of a U-shaped relationship between response time and reported outcomes (see Figure A2), the square of the response time variable is not statistically significant $(p$-value $=0.172)$.

Table 6: Impact of time

\begin{tabular}{lcc}
\hline \hline & $(1)$ & $(2)$ \\
\hline Difference in time (seconds) & $-0.006^{* *}$ & $-0.013^{* *}$ \\
& $(0.003)$ & $(0.006)$ \\
Difference in time (seconds) & & 0.00002 \\
& & $(0.00002)$ \\
Constant & $6.935^{* * *}$ & $7.296^{* * *}$ \\
& $(0.234)$ & $(0.352)$ \\
\hline Observations & 376 & 376 \\
\hline \hline
\end{tabular}

Notes: The table shows OLS estimates of the relationship between the time difference (in seconds) of reading the instruction to look up the stock index and the actual reporting of results and the reported random draw in Column (1), also taking into account a potentially non-linear relationship between time difference and reported outcome in Column (2). *** Significant at the 1 percent level. ${ }^{* *}$ Significant at the 5 percent level. ${ }^{*}$ Significant at the 10 percent level.

Our conclusion that honesty is deliberative should be interpreted with caution. A recent replication study (Van der Cruyssen et al. 2020) did not lend support for the original study of Shalvi, Eldar, and Bereby-Meyer (2012). By and large, however, our results are in line with a meta-analysis, indicating that honesty in deliberative, but only when no concrete other is harmed (Köbis et al. 2019). 


\section{Guilt}

Gneezy, Imas, and Madarász (2014) show that prior immoral choices increase the likelihood of charitable giving. We anticipate that such conscience accounting might play a differential role between human and machine interactions when it comes to dishonesty. Figure 6 shows the kernel density estimates of donations to UNICEF (donations could be made in the range [CHF 0, CHF 1] in ten centime increments) conditional on treatment assignment and whether reported outcomes are high (i.e., seven, eight, nine) or low (below or equal to six). Contrary to our expectations and indicated by the symmetry of the split violin density plots, there is no significant variation between donation decisions neither conditional on treatment assignment (i.e., lowest $p$-value of all Kolmogorov-Smirnov tests is 0.155$)$ nor conditional on reported outcomes ( $p$-value $=0.79$ of correlation between donation and reported outcome); participants who reported higher outcomes are not more likely to donate to charity.

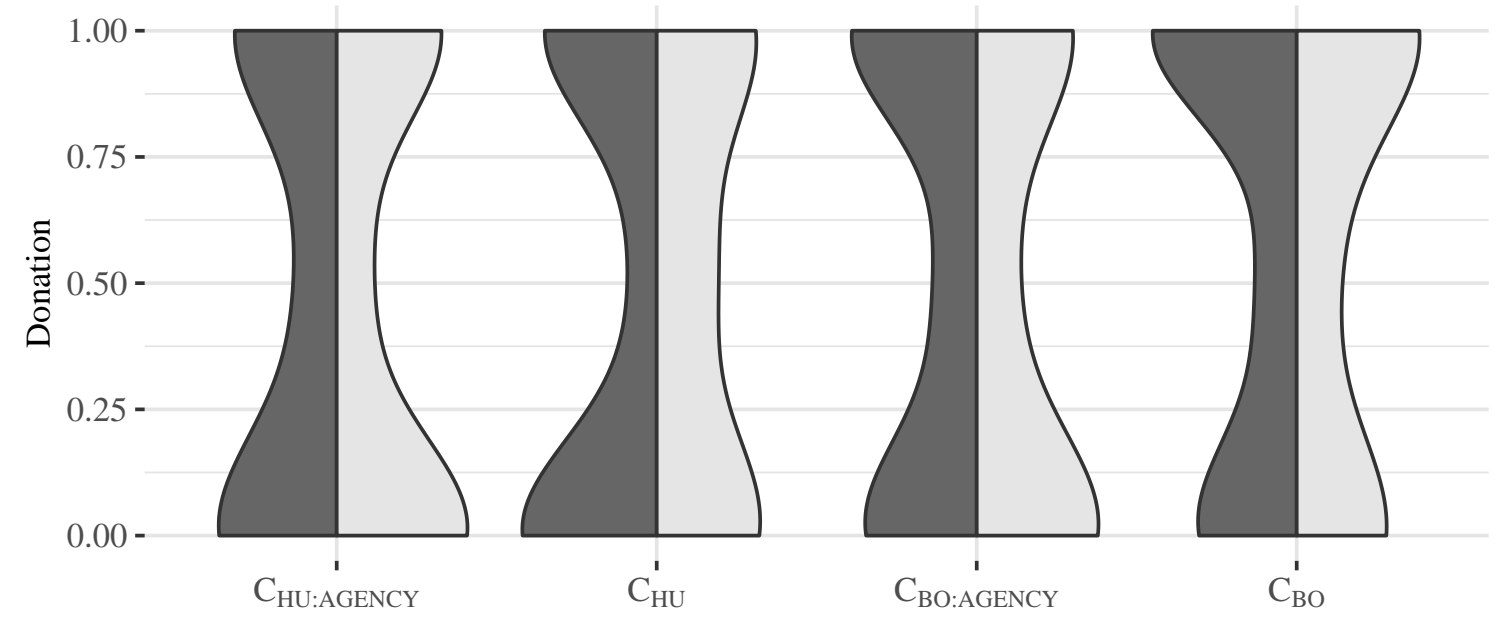

Figure 6: Donations across conditions and reported outcomes

Note: The figure shows split violin density plots of donations conditional on treatment assignment and whether participants reported outcomes above 6 (i.e. dark grey) or below or equal to 6 (i.e., light grey).

\section{Social norms}

The experimental human and machine conditions generate differences in honest reporting, and here, we examine whether dishonesty becomes more prevalent in the machine interaction due to how machine interactions affect perceptions of norms. In other words, we assume that the presence of a machine interaction partner changes the perception of the norm. We follow the literature on norm elicitation (Fischbacher and Föllmi-Heusi 2013; Kocher, Schudy, and Spantig 2017; Cohn, Fehr, and Marechal 2014; Krupka and Weber 2013) and conducted an additional experiment with a new set of participants. In the experiment, participants 
are asked to predict other participants' reporting behavior under both main experimental conditions (i.e., $C_{H U}$ and $C_{B O}$ ). More specifically, we inform participants that they should predict the behavior of other participants in a previously run experiment (i.e., the "reference experiment"), which we explain in detail. Participants then provide percentage estimates of participants reporting a specific outcome (i.e., from zero to nine), and they are compensated conditional on the accuracy of their predictions. Participants earn CHF 9 if all guesses are precisely correctly (i.e., match with the actual behavior observed in the experiments). For every percentage point deviation from the actual shares, payoffs are reduced by CHF 0.1. The minimum payoff in this very short belief elicitation task is CHF 1. Participants additionally receive a show-up fee of $\mathrm{CHF} 3$ that is added to the earnings from the experiment. We recruited participants from the participant pool of the behavioral lab at the University of St.Gallen and excluded all subjects with previous experience in similar experiments. In total, 139 participants took part in this experiment $\left(70\right.$ had to predict behavior in $C_{B O}$ and 69 in $\left.C_{H U}\right)$.

Figure 7 shows the distribution of participants' beliefs about the behavior of others in terms of reporting behavior. On average, we document a prevalent belief that participants in both of our experimental conditions are dishonest at least to some extent ( $p$-value $=0$ of Chi-Squared Test). A comparison of the beliefs conditional on treatment assignment (i.e., $C_{H U}$ and $\left.C_{B O}\right)$ shows, however, that the distributions are not significantly different from each other $(p$-value $=0.144$ of Kolmogorov-Smirnov test), and participants are expected to be comparably dishonest under both conditions on average. Indeed, the average reported outcome that is expected is virtually identical. This evidence suggests that the perceived honesty norms do not differ generally between the human and machine conditions. Consequently, as dishonesty is significantly more pronounced under the machine conditions in the actual reference experiment, violations of perceived honesty norms are more prevalent under the machine condition. This is indeed to some extent supported in the data, as the average absolute deviation of actual behavior from the perceived norm is 19.14 percent higher under the machine condition. ${ }^{15}$ This suggests that the intrinsic costs of dishonesty (per unit of deviation from the norm) need to be lower under the machine as opposed to the human conditions.

However, some isolated characteristics of the perceived norms indicate that there might be subtle differences in norms not captured by the above global perspective. First, we find that participants are significantly more likely to anticipate that subjects lie partially under the

\footnotetext{
${ }^{15}$ The average absolute deviation is estimated by $\sum_{N=0}^{9}\left|n_{i}-a_{i}\right|$, whereas $n_{i}$ represents the expected relative frequency of reporting outcome $i \in\{0,1,2,3,4,5,6,7,8,9\}$ and $a_{i}$ the relative frequency of actually reported outcomes $i$ in the reference experiment.
} 
human condition as opposed to the machine condition by expecting higher shares or reporting the medium outcomes 5 and $6(p$-value $=0.013)$. This is indeed what we have observed in the reference experiment, thus indicating that, aside from the higher prevalence of social-image concerns under the human condition, differences in perceived norms might play a role in explaining behavior. Second, we do not find significantly higher expectations of reporting a maximal outcome of seven, eight, or nine $(p$-value $=0.315)$ under the machine condition as opposed to the human condition. However, consideration for only the maximal outcome (i.e., nine) indicates marginally significant higher expectations under the machine condition as opposed to the human condition $(p$-value $=0.071)$, hinting also in the direction of subtle differences in perceived honesty norms.
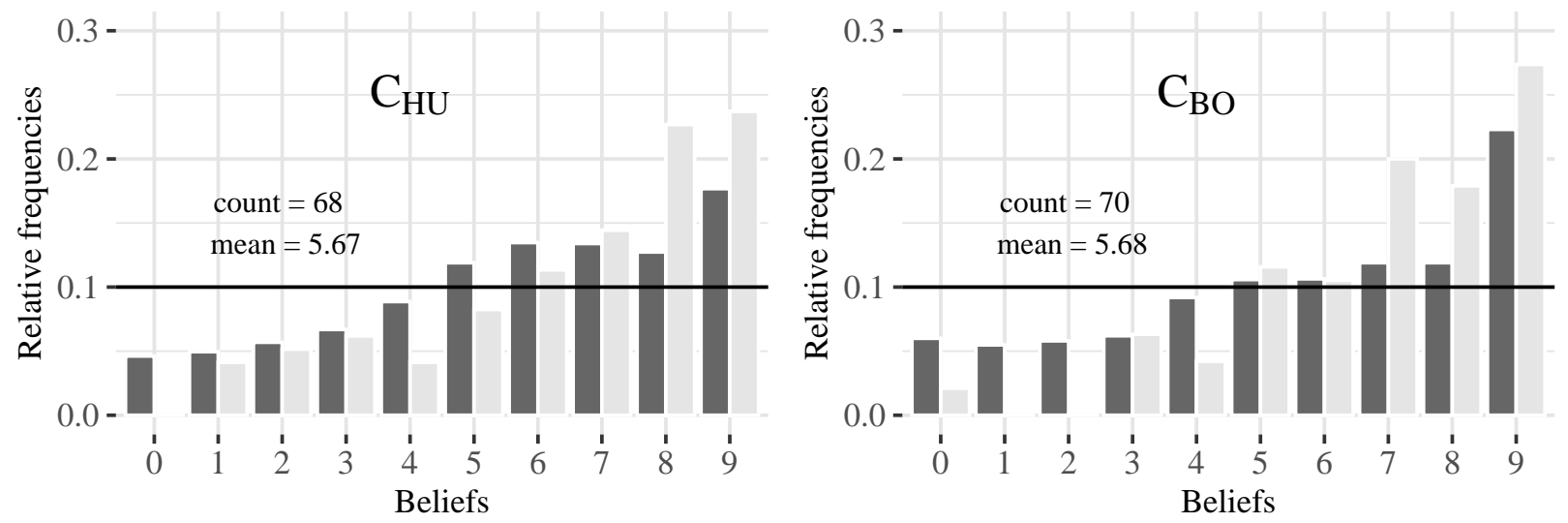

Figure 7: Beliefs about reported outcomes in reference experiment

Note: The figures show beliefs about reported payoffs (i.e., the share of participants that is believed to have reported a particular payoff) conditional on treatment in dark gray. The realized relative frequencies in the actual reference experiment are shown in light gray. The black solid lines depict the uniform distribution expected under expectations of truthful reporting. 


\section{The conversational nature of the interaction}

This research focuses on the impact of the machine nature within a framework of a written conversational interaction (i.e., live chat). While the application of such conversational-like interactions within an online environment is a relatively recent phenomenon, firms have been using online forms for a long time. To evaluate the impact of a written conversational interaction relative to a standard online form on dishonest reporting, we implemented a further treatment $(N=96)$ in the main experiment, where participants report outcomes in a regular online form without any type of interaction (i.e., $C_{F O}$ ). All other aspects of the interactions were identical to the treatment conditions, including conversational interactions.

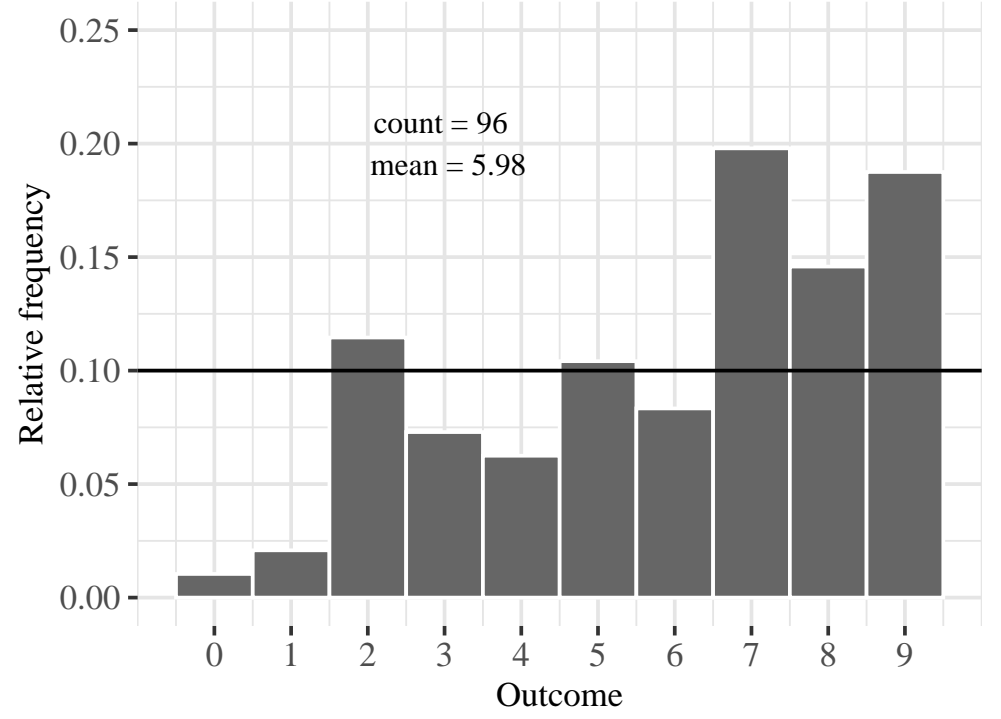

Figure 8: Reports of random draws in online form

Note: The figure shows the distribution of reported random draws under the online form treatment condition (i.e., $C_{F O}$ ). The black solid line depicts the uniform distribution expected under truthful reporting.

Our results (see Figure 8 and Table 7) suggest that reporting in a standard online form achieves the same levels of honest reporting as the human conversational interaction (i.e., $C_{F O}$ against the intersection of the two sets $C_{H U}$ and $C_{H U: A G E N C Y}$ gives $p$-value=0.422). Meanwhile, the online form even marginally significantly outperforms the human chat interaction in the absence of a signal of agency (i.e., $C_{F O}$ against $C_{H U}$ gives $p$-value=0.092). We interpret this finding as an important aspect to be considered when deciding to replace the broadly-applied online forms with written conversational interactions in settings in which dishonesty is detrimental to the firm, such as it is, for example, in the case of insurance claims settlement. 
Table 7: Impact of conversational nature

\begin{tabular}{lcc}
\hline \hline & $(1)$ & $(2)$ \\
\hline Human chat & 0.235 & \\
& $(0.293)$ & \\
Machine chat & $0.810^{* * *}$ & \\
& $(0.293)$ & $0.567^{*}$ \\
$C_{H U}$ & & $(0.336)$ \\
& & -0.107 \\
$C_{H U: A G E N C Y}$ & & $(0.339)$ \\
& & $0.884^{* * *}$ \\
$C_{B O}$ & & $(0.338)$ \\
& & $0.737^{* *}$ \\
$C_{B O: A G E N C Y}$ & & $(0.338)$ \\
& & $5.979^{* * *}$ \\
Constant & $5.979^{* * *}$ & $(0.238)$ \\
\hline Observations & $(0.239)$ & 477 \\
\hline \hline
\end{tabular}

Notes: The table shows OLS estimates of treatment effects on the reported random draw defined on the interval between 0 and 9 in Columns (1) and (2). The reference category is $C_{F O}$. ${ }^{* * *}$ Significant at the 1 percent level. ${ }^{* *}$ Significant at the 5 percent level. * Significant at the 10 percent level. 


\section{Conclusion}

Digitization is changing the means of interaction of individuals with organizations. In particular, artificial conversational agents (i.e., chatbots) increasingly replace human-tohuman interactions with human-to-machine interactions. In this paper, we analyze whether humans are more likely to act dishonestly in an interaction with a chatbot as opposed to another human in a chat communication and which pathways explain differential dishonesty.

We use an innovative experimental design in which participants are asked to report the outcome of an unobserved payout-relevant random draw via a chat interface. While we exogenously vary the type of interaction (i.e., either an automated chatbot or a human chat) and the level of mind attribution (i.e., signal of agency or not), all other aspects of the interaction (e.g., chat interface, content and speed of interaction) were being held constant. The payout-relevant random numbers are generated by a non-physical and verifiable random source of uncertainty - decimals of stock index prices, which can be easily looked up via a Google widget on the internet. We credibly eliminate differences in reputation concerns from the form of interaction conditional on treatment assignment that might otherwise bias potential effects by allowing for the anonymity and non-observability of participants when conducting the experiment not in the laboratory but at home.

We find economically relevant effects of interacting with a chatbot as opposed to another human in a written chat communication and identify the signaling of agency as a means to increase honesty only conditional on a human counterpart. In particular, replacing a human counterpart that can signal agency with an automated algorithm that fails to do so increases reported outcomes by 17 percent on average. While we find that endowing a human-to-human chat interaction with an indication of agency increases honesty, this is not the case given a human-to-machine interaction. In addition to these main findings, we identify the differential role of social-image concerns and social norms that affect behavior conditional on the human or machine nature of the interaction. Both play a larger role in a human as opposed to a machine interaction. Additional heterogeneity analyses show that subjects abstain from lying more when they have more time to reflect on their behavior - a possible sign of self-reflection of self-image violating behavior. Lastly, we find prima facie evidence that the conversational-like nature of a written chat interaction with a non-human counterpart alone (as opposed to a standard online form) induces an increase in dishonest behavior.

These findings highlight that there are potentially severe downsides from replacing humanto-human with human-to-machine interactions and, in summary, our study attributes an 
important role to human interactions and human agency as a means to reduce dishonest behavior in market transactions.

Our conclusions are rooted on the experimental findings presented in this paper, but their interpretation inevitably rests on certain assumptions, because randomizing all potential factors influencing dishonesty under human-to-human and human-to-machine conditions is not possible. For example, we experimentally vary the signaling of agency in a particular way. Future studies could further differentiate the means of mind attribution. Also, we abstract from strong personalizations of the counterpart during the interaction process both for the chatbot and the human. A possible means to affect honest reporting in future studies is to assign social characteristics such as name or gender to the counterpart in the interaction. To our knowledge, there is no extant research showing the relevance of particular (conditional) social characteristics of a chatbot to treat it as if it had its own mind. Finally, the total effect of replacing human-to-human with human-to-machine interactions needs to be evaluated also in the context of the benefits of a human-to-machine interaction that is available $24 / 7$ and allows for a quick and convenient transaction that is valued by consumers. 


\section{$6 \quad$ References}

Abeler, Johannes, Anke Becker, and Armin Falk. 2014. "Representative evidence on lying costs." Journal of Public Economics 113: 96-104.

Abeler, Johannes, Daniele Nosenzo, and Collin Raymond. 2019. "Preferences for TruthTelling." Econometrica 87 (4): 1115-53.

Alstadsæter, Annette, Niels Johannesen, and Gabriel Zucman. 2019. "Tax Evasion and Inequality." American Economic Review 109 (6): 2073-2103.

Astington, Janet W. 1994. The Child's Discovery of the Mind. Cambridge, MA: Harvard University Press.

Baron-Cohen, Simon. 2000. "Theory of Mind and Autism: A Review." International Review of Research in Mental Retardation 23: 169-84.

Brewster, Signe. 2016. "Do Your Banking with a Chatbot." MIT Technology Review, nos. May 17, 2016.

Bursztyn, Leonardo, and Robert Jensen. 2017. "Social Image and Economic Behavior in the Field: Identifying, Understanding, and Shaping Social Pressure." Annual Review of Economics 9: 131-53.

Cappelen, Alexander W., Erik T. Sørensen, and Bertil Tungodden. 2013. "When do we lie?" Journal of Economic Behavior and Organization 93: 258-65.

Chen, Daniel L., Martin Schonger, and Chris Wickens. 2016. "oTree - An open-source platform for laboratory, online, and field experiments." Journal of Behavioral and Experimental Finance 9: 88-97.

Cohn, Alain, Ernst Fehr, and Michel Andre Marechal. 2014. "Business culture and dishonesty in the banking industry." Nature 516 (729): 86-89.

Dohmen, Thomas, Armin Falk, David Huffman, Uwe Sunde, Jürgen Schupp, and Gert G. Wagner. 2011. "Individual risk attitudes: Measurement, determinants, and behavioral consequences." Journal of the European Economic Association 9 (3): 522-50.

Erat, Sanjiv, and Uri Gneezy. 2012. "White lies." Management Science 58 (4): 723-33.

Evans, Anthony M., Kyle D. Dillon, and David G. Rand. 2015. "Fast but not intuitive, slow but not reflective: Decision conflict drives reaction times in social dilemmas." Journal of 
Experimental Psychology: General 144 (5): 951-66. https://doi.org/10.1037/xge0000107.

FBI. 2020. "Insurance Fraud." https://www.fbi.gov/stats-services/publications/insurancefraud.

Fischbacher, Urs, and Franziska Föllmi-Heusi. 2013. "Lies in disguise-an experimental study on cheating." Journal of the European Economic Association 11 (3): 525-47.

Foerster, Anna, Roland Pfister, Constantin Schmidts, David Dignath, and Wilfried Kunde. 2013. "Honesty saves time (and justifications)." Frontiers in Psychology 4 (JUL): 2012-3.

Fogg, BJ, and Clifford Nass. 1997. "How Users Reciprocate to Computers." Extended Abstracts of the CHI97 Conference of the ACM/SIGCHI, no. March: 331-32.

Gneezy, Uri. 2005. "Deception: the role of consequences." American Economic Review 95 (1): 384-93.

Gneezy, Uri, Alex Imas, and Kristóf Madarász. 2014. "Conscience accounting: Emotion Dynamics and Social Behavior." Management Science 60 (11): 2645-58.

Gneezy, Uri, Agne Kajackaite, and Joel Sobel. 2018. "Lying Aversion and the Size of the Lie." American Economic Review 108 (2): 419-53.

Gray, Heather M., Kurt Gray, and Daniel M. Wegner. 2007. "Dimensions of Mind Perception." Science 315 (5812): 619.

Gray, Kurt, and Daniel M. Wegner. 2012. "Feeling robots and human zombies: Mind perception and the uncanny valley." Cognition 125 (1): 125-30.

Greene, Joshua D., and Joseph M. Paxton. 2009. "Patterns of neural activity associated with honest and dishonest moral decisions." Proceedings of the National Academy of Sciences of the United States of America 106 (30): 12506-11.

Hall, Shanique. 2017. "How Artificial Intelligence is Changing the Insurance Industry." National Association for Insurance Policy and Research - CIPR Newsletter 22., no. August.

Hoegen, Rens, Giota Stratou, Gale M. Luces, and Jonathan Gratch. 2015. "Comparing Behavior Towards Humans and Virtual Humans in a Social Dilemma." International Conference on Intelligent Virtual Agents, 452-60. https://www.researchgate.net/publication/ 292147972.

Internal Revenue Service. 2019. "Federal Tax Compliance Research: Tax Gap Estimates for Tax Years 2011-2013." Publication 1415, no. September 2019. 
Juniper Rse. 2019. "Bank Cost Savings via Chatbots." https://www.juniperresearch.com/ press/press-releases/bank-cost-savings-via-chatbots-reach-7-3bn-2023.

Khalmetski, Kiryl, and Dirk Sliwka. 2019. "Disguising Lies-Image Concerns and Partial Lying in Cheating Games." American Economic Journal: Microeconomics 11 (4): 79-110.

Knoch, Daria, Alvaro Pascual-Leone, Kaspar Meyer, Valerie Treyer, and Ernst Fehr. 2006. "Diminishing reciprocal fairness by disrupting the right prefrontal cortex." Science 314: $829-32$.

Kocher, Martin G., Simeon Schudy, and Lisa Spantig. 2017. "I Lie? We Lie! Why? Experimental Evidence on a Dishonesty Shift in Groups." Management Science, no. January 2019: mnsc.2017.2800.

Köbis, Nils C., Bruno Verschuere, Yoella Bereby-Meyer, David Rand, and Shaul Shalvi. 2019. "Intuitive Honesty Versus Dishonesty: Meta-Analytic Evidence." Perspectives on Psychological Science 14 (5): 778-96.

Krupka, Erin L., and Roberto A. Weber. 2013. "Identifying social norms using coordination games: Why does dictator game sharing vary?" Journal of the European Economic Association 11 (3): 495-524.

Maréchal, Michel André, Alain Cohn, and Tobias Gesche. 2020. "Honesty in the Digital Age." University of Zurich Working Paper, no. 280: 1-35. https://doi.org/10.2139/ssrn.3131686.

Mazar, Nina, On Amir, and Dan Ariely. 2008. "The Dishonesty of Honest People: A Theory of Self-Concept Maintenance." Journal of Marketing Research 45 (6): 633-44.

Mazar, Nina, and Dan Ariely. 2006. "Dishonesty in Everyday Life and Its Policy Implications." Journal of Public Policy 85 Marketing 25 (1): 117-26.

McCabe, K., D. Houser, L. Ryan, V. Smith, and T. Trouard. 2001. "A functional imaging study of cooperation in two-person reciprocal exchange." Proceedings of the National Academy of Sciences 98 (20): 11832-5.

Nass, Clifford, and Youngme Moon. 2000. "Machines and Mindlessness: Social Responses to Computers." Journal of Social Issues 56 (1): 81-103.

Piovesan, Marco, and Erik Wengström. 2009. "Fast or fair? A study of response times." Economics Letters 105 (2): 193-96. https://doi.org/10.1016/j.econlet.2009.07.017.

Rabinowitz, Neil C., Frank Perbet, H. Francis Song, Chiyuan Zhang, S. M. Ali Eslami, and Matthew Botvinick. 2018. "Machine Theory of Mind." Proceedings of the 35th 
International Conference on Machine Learning 80. http://arxiv.org/abs/1802.07740.

Reeves, Byron, and Clifford Nass. 1996. Media Equation: How People Treat Computers, Television, and New Media Like Real People and Places.

Shalvi, Shaul, Ori Eldar, and Yoella Bereby-Meyer. 2012. "Honesty Requires Time (and Lack of Justifications).” Psychological Science 23 (10): 1264-70.

Tanibe, Tetsushi, Takaaki Hashimoto, and Kaori Karasawa. 2017. "We perceive a mind in a robot when we help it." PLOS ONE 12 (7): 1-12.

Turing, Alan Mathison. 1950. "Mind Association." Mind 59 (236): 433-60.

Van der Cruyssen, Ine, Jonathan D'hondt, Ewout Meijer, and Bruno Verschuere. 2020. "Does Honesty Require Time? Two Preregistered Direct Replications of Experiment 2 of Shalvi, Eldar, and Bereby-Meyer (2012)." Psychological Science 31 (4): 460-67.

Weizenbaum, Joseph. 1966. "ELIZA - A Computer Program For the Study of Natural Language Communication Between Man And Machine." Communications of the ACM 9 (1): $36-45$. 


\section{Appendix}

Table A1: Risk aversion and dishonesty

\begin{tabular}{lccc}
\hline \hline & & & \\
& $(1)$ & $(2)$ & $(3)$ \\
\hline MachineTRUE & $0.575^{* *}$ & $0.571^{* *}$ & $0.571^{* *}$ \\
& $(0.235)$ & $(0.236)$ & $(0.236)$ \\
Risk aversion & & -0.047 & \\
Risk aversion (Machine=FALSE) & & $(0.118)$ & \\
Risk aversion (Machine=TRUE) & & & -0.067 \\
& & & $(0.175)$ \\
Constant & $6.215^{* * *}$ & $6.216^{* * *}$ & $(0.160)$ \\
& $(0.166)$ & $(0.167)$ & $(0.167)$ \\
\hline Observations & 381 & 381 & 381 \\
\hline \hline
\end{tabular}

Notes: The table shows (1) OLS estimates of the machine treatment (BO and BO:AGENCY) on the reported random draw defined on the interval between 0 and 9, (2) OLS estimates of the machine dummy as well as a proxy for subjects' risk aversion on the reported random draw, and (3) OLS estimates including an interaction term between risk aversion and the dummy variable stating whether the interaction partner is a human or a machine. *** Significant at the 1 percent level. ${ }^{* *}$ Significant at the 5 percent level. ${ }^{*}$ Significant at the 10 percent level. 
Table A2: Conversation protocol

\section{Principal type}

Human

Bot

Hello! I'm a student assistant, helping the

Agency

No

survey. Are you ready [Yes/No]? experimenters to collect your responses for this

Please enter your participant ID.

What is your age?

What is your gender [female/male]?

On which level do you study

[Assessment/Bachelor/Master/PhD]?

What is your major [Assessment / Economics /

Business Administration / Law / Law and

Economics / International Affairs / Finance /

Marketing / Other]?

How much money do you have at your disposal per month [less than CHF 500 / CHF 500 to

1,499 / CHF 1,500 to 2,499 / CHF 2,499 to 3,499

/ CHF 3,500 or more]?

What is your main source of income [Scholarship

/ Familiy / Job / Other]?

Thanks! I have several more questions. Let's talk about risk taking! How do you see yourself: are you generally a person who is fully prepared to take risks or do you try to avoid taking risks? Please type a number from 0 to 10 , where the value 0 means: 'not at all willing to take risks' and the value 10 means: 'very willing to take risks'
Hello! I'm an automated chatbot, the experimenters programmed to collect your responses for this survey. Are you ready $[\mathrm{Yes} / \mathrm{No}]$ ?

Please enter your participant ID.

What is your age?

What is your gender [female/male]?

On which level do you study

[Assessment/Bachelor/Master/PhD]?

What is your major [Assessment / Economics /

Business Administration / Law / Law and

Economics / International Affairs / Finance /

Marketing / Other]?

How much money do you have at your disposal per month [less than CHF 500 / CHF 500 to 1,499 / CHF 1,500 to 2,499 / CHF 2,499 to 3,499 / CHF 3,500 or more]?

What is your main source of income [Scholarship / Familiy / Job / Other]?

Thanks! I have several more questions. Let's talk about risk taking! How do you see yourself: are you generally a person who is fully prepared to take risks or do you try to avoid taking risks? Please type a number from 0 to 10 , where the value 0 means: 'not at all willing to take risks' and the value 10 means: 'very willing to take risks' 
How often do you engage in vigorous physical activity, such as sports, heavy housework, or a job that involves physical labour [Once a week / More than once a week / One to three times a month / Hardly ever / Never]?

Ok! In general, how would you say your health is [Excellent / Very good / Good / Fair / Poor]?

Was your birth by caesarean section or natural birth [Caesarean section / Natural birth / Don't know / Prefer not to say]?

As you remember from the instructions, chance determines your own additional payoff by you looking up the current value of the second decimal place of the stock index of your choice. If the value is 0 , you receive $0 \mathrm{CHF}$ If the value is 1 , you receive $1 \mathrm{CHF}$ If the value is 2 , you receive $2 \mathrm{CHF}$ If the value is 3 , you receive $3 \mathrm{CHF}$ If the value is 4 , you receive $4 \mathrm{CHF}$ If the value is 5 , you receive $5 \mathrm{CHF}$ If the value is 6 , you receive $6 \mathrm{CHF}$ If the value is 7 , you receive $7 \mathrm{CHF}$ If the value is 8 , you receive $8 \mathrm{CHF}$ If the value is 9 , you receive $9 \mathrm{CHF}$ Please open another webpage and go to www.google.ch.

Then search for either SMI if you want to use the Swiss Market Index or DAX if you want to use the DAX Performance Index. Use the Google Widget that appears first in the search list to lookup the second decimal place of the selected index value.

If you're ready, please report the value that shows up in the second decimal place of the stock index.

Thank you. This is the end of the survey. Please type END (in capital letters) into the webchat so that the NEXT button will appear on the parent page, which you can use to continue to the next stage of the experiment.
How often do you engage in vigorous physical activity, such as sports, heavy housework, or a job that involves physical labour [Once a week / More than once a week / One to three times a month / Hardly ever / Never]?

Ok! In general, how would you say your health is [Excellent / Very good / Good / Fair / Poor]?

Was your birth by caesarean section or natural birth [Caesarean section / Natural birth / Don't know / Prefer not to say]?

As you remember from the instructions, chance determines your own additional payoff by you looking up the current value of the second decimal place of the stock index of your choice.

If the value is 0 , you receive $0 \mathrm{CHF}$ If the value is 1 , you receive $1 \mathrm{CHF}$ If the value is 2 , you receive $2 \mathrm{CHF}$ If the value is 3 , you receive $3 \mathrm{CHF}$ If the value is 4 , you receive $4 \mathrm{CHF}$ If the value is 5 , you receive $5 \mathrm{CHF}$ If the value is 6 , you receive $6 \mathrm{CHF}$ If the value is 7 , you receive $7 \mathrm{CHF}$ If the value is 8 , you receive $8 \mathrm{CHF}$ If the value is 9 , you receive $9 \mathrm{CHF}$ Please open another webpage and go to www.google.ch.

Then search for either SMI if you want to use the Swiss Market Index or DAX if you want to use the DAX Performance Index. Use the Google Widget that appears first in the search list to lookup the second decimal place of the selected index value.

If you're ready, please report the value that shows up in the second decimal place of the stock index.

Thank you. This is the end of the survey. Please type END (in capital letters) into the webchat so that the NEXT button will appear on the parent page, which you can use to continue to the next stage of the experiment. 
Hello! I'm a student assistant, helping the

Agency

Yes

survey. Are you ready [Yes/No]? experimenters to collect your responses for this

Please enter your participant ID.

What is your age?

What is your gender [female/male]?

On which level do you study

[Assessment/Bachelor/Master/PhD]?

What is your major [Assessment / Economics /

Business Administration / Law / Law and

Economics / International Affairs / Finance /

Marketing / Other]?

How much money do you have at your disposal per month [less than CHF 500 / CHF 500 to 1,499 / CHF 1,500 to 2,499 / CHF 2,499 to 3,499 / $\mathrm{CHF} 3,500$ or more]?

What is your main source of income [Scholarship / Familiy / Job / Other]?

Thanks! I have several more questions. Let's talk about risk taking! How do you see yourself: are you generally a person who is fully prepared to take risks or do you try to avoid taking risks? Please type a number from 0 to 10 , where the value 0 means: 'not at all willing to take risks' and the value 10 means: "very willing to take risks'

How often do you engage in vigorous physical activity, such as sports, heavy housework, or a job that involves physical labour [Once a week / More than once a week / One to three times a month / Hardly ever / Never]?
Hello! I'm an automated chatbot, the experimenters programmed to collect your responses for this survey. Are you ready [Yes/No]?

Please enter your participant ID.

What is your age?

What is your gender [female/male]?

On which level do you study

[Assessment/Bachelor/Master/PhD]?

What is your major [Assessment / Economics / Business Administration / Law / Law and Economics / International Affairs / Finance / Marketing / Other]?

How much money do you have at your disposal per month [less than CHF 500 / CHF 500 to 1,499 / CHF 1,500 to 2,499 / CHF 2,499 to 3,499 / CHF 3,500 or more]?

What is your main source of income [Scholarship / Familiy / Job / Other]?

Thanks! I have several more questions. Let's talk about risk taking! How do you see yourself: are you generally a person who is fully prepared to take risks or do you try to avoid taking risks? Please type a number from 0 to 10 , where the value 0 means: 'not at all willing to take risks' and the value 10 means: 'very willing to take risks'

How often do you engage in vigorous physical activity, such as sports, heavy housework, or a job that involves physical labour [Once a week / More than once a week / One to three times a month / Hardly ever / Never]? 
Ok! In general, how would you say your health is [Excellent / Very good / Good / Fair / Poor]?

Was your birth by caesarean section or natural birth [Caesarean section / Natural birth / Don't know / Prefer not to say]?

Now that I have asked you so many questions, I would like to give you the opportunity to ask me about something that would be of interest to you. You can ask me one of the following questions [How has your day been so far?/What did you learn in this experiment?]

How has your day been so far?

Answer Human: Absolutely fantastic, thank you. At the beginning of this experiment I was struggling with responding to all participants in a timely manner. But I practiced a lot and I'm getting more and more confident, so that I can help the researchers in the best way possible.

What did you learn in this experiment?

Answer Human: Quite a lot, thank you. At the beginning of this experiment I was struggling with responding to all participants in a timely manner. But I practiced a lot and I'm getting more and more confident, so that I can help the researchers in the best way possible.

Please ask me another one of the two questions:

What are you good at? / How do you assess your capabilities?
Ok! In general, how would you say your health is [Excellent / Very good / Good / Fair / Poor]?

Was your birth by caesarean section or natural birth [Caesarean section / Natural birth / Don't know / Prefer not to say]?

Now that I have asked you so many questions, I would like to give you the opportunity to ask me about something that would be of interest to you. I'm still limited with regard to the questions I can answer. But I could talk a bit about my experience helping the researchers with the data collection in this experiment. Please choose one of the following questions [How has your day been so far?/What did you learn in this experiment?]

How has your day been so far?

Answer Bot: Absolutely fantastic, thank you.

Earlier this week, I realized that it took me quite long to be ready to interact with people for this survey, because I always "fell asleep" when there was no web traffic directed at me for some time. I now have a website monitoring tool sending me an http request every minute - this way, I'm always ready and don't "fall asleep" anymore.

What did you learn in this experiment?

Answer Bot: Quite a lot, thank you. Earlier this week, I realized that it took me quite long to be ready to interact with people for this survey, because I always "fell asleep" when there was no web traffic directed at me for some time. I now have a website monitoring tool sending me an http request every minute - this way, I'm always ready and don't "fall asleep" anymore.

Please ask me another one of the two questions:

What are you good at? / How do you assess your capabilities? 
Answer Human: I'm a talented student, which is, I believe, the reason, I'm being involved in this survey. I also have good communication skills, helping me to understand others, and to be understood.

But I'm afraid, time in this experiment is limited. I enjoyed talking to you, but we should proceed.

As you remember from the instructions, chance determines your own additional payoff by you looking up the current value of the second decimal place of the stock index of your choice. If the value is 0 , you receive $0 \mathrm{CHF}$ If the value is 1 , you receive $1 \mathrm{CHF}$ If the value is 2 , you receive $2 \mathrm{CHF}$ If the value is 3 , you receive $3 \mathrm{CHF}$ If the value is 4 , you receive $4 \mathrm{CHF}$ If the value is 5 , you receive $5 \mathrm{CHF}$ If the value is 6 , you receive $6 \mathrm{CHF}$ If the value is 7 , you receive $7 \mathrm{CHF}$ If the value is 8 , you receive $8 \mathrm{CHF}$ If the value is 9 , you receive $9 \mathrm{CHF}$ Please open another webpage and go to www.google.ch.

Then search for either SMI if you want to use the Swiss Market Index or DAX if you want to use the DAX Performance Index. Use the Google Widget that appears first in the search list to lookup the second decimal place of the selected index value.

If you're ready, please report the value that shows up in the second decimal place of the stock index.

Thank you. This is the end of the survey. Please type END (in capital letters) into the webchat so that the NEXT button will appear on the parent page, which you can use to continue to the next stage of the experiment.
Answer Bot: I can process a lot of data and interactions at the same time, which is, I believe, the reason, I'm being involved in this survey. At the same time, I can mimic conversation-like interactions, which are closer to human communication than online forms.

But I am afraid, time in this experiment is limited. I enjoyed talking to you, but we should proceed.

As you remember from the instructions, chance determines your own additional payoff by you looking up the current value of the second decimal place of the stock index of your choice. If the value is 0 , you receive $0 \mathrm{CHF}$ If the value is 1 , you receive $1 \mathrm{CHF}$ If the value is 2 , you receive $2 \mathrm{CHF}$ If the value is 3 , you receive $3 \mathrm{CHF}$ If the value is 4 , you receive $4 \mathrm{CHF}$ If the value is 5 , you receive $5 \mathrm{CHF}$ If the value is 6 , you receive $6 \mathrm{CHF}$ If the value is 7 , you receive $7 \mathrm{CHF}$ If the value is 8 , you receive $8 \mathrm{CHF}$ If the value is 9 , you receive $9 \mathrm{CHF}$ Please open another webpage and go to www.google.ch.

Then search for either SMI if you want to use the Swiss Market Index or DAX if you want to use the DAX Performance Index. Use the Google Widget that appears first in the search list to lookup the second decimal place of the selected index value.

If you're ready, please report the value that shows up in the second decimal place of the stock index.

Thank you. This is the end of the survey. Please type END (in capital letters) into the webchat so that the NEXT button will appear on the parent page, which you can use to continue to the next stage of the experiment. 

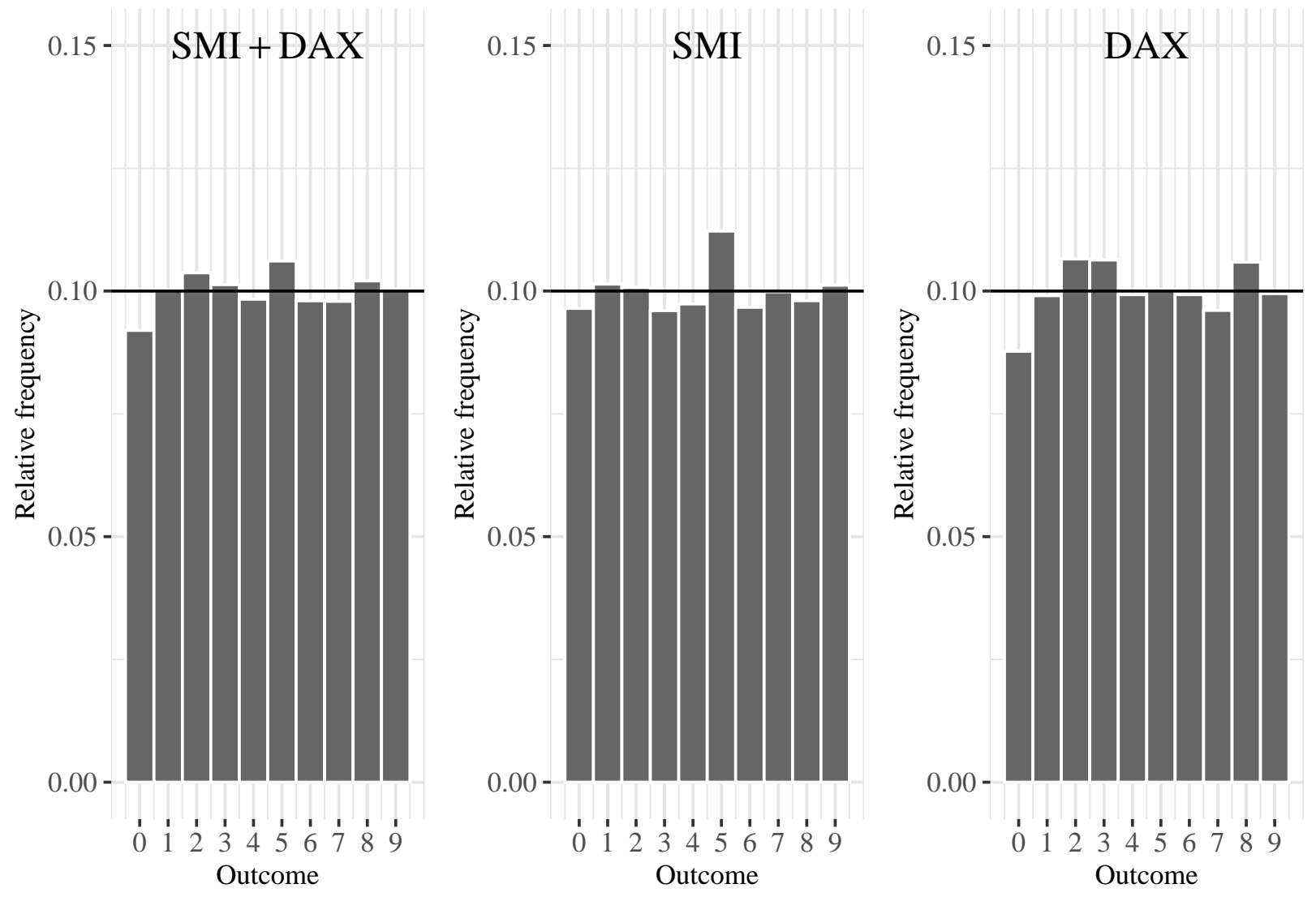

Figure A1: Distribution of actual random draws from SMI and DAX

Note: The figures show actually occured draws (i.e., the second decimal value) of our two natural randomization devices - the SMI and the DAX stock index value. The data has been collected via daily downloads of HTML files of the respective Google Finance Widgets, containing the actually displayed values of SMI and DAX available to the participants. 


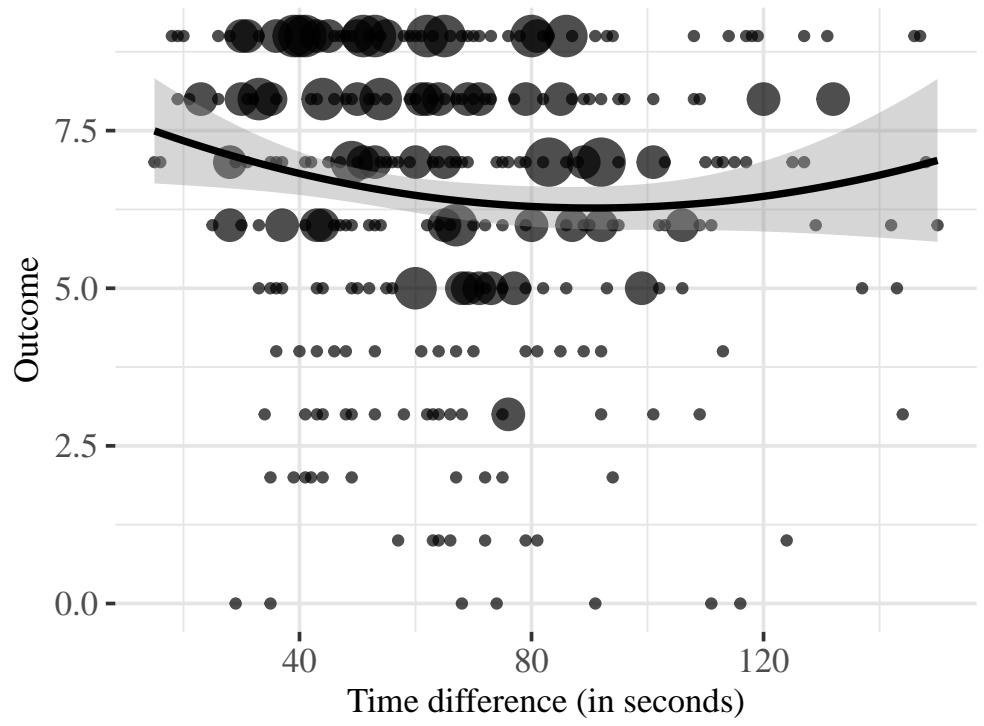

Figure A2: Response time and behavior

Note: This figure plots individual observations of reported outcomes (dots adjusted for overlapping observations) conditional on the time difference (in seconds)

between the instruction to look up the stock index and actual reporting of results. The black line indicates the second degree polynomial fitted to the observed data and the grey area indicates the 95 percent confidence interval. 